\title{
An intermediate animal model of spinal cord stimulation
}

\author{
Thomas Guiho (1), Christine Azevedo Coste (1), Claire Delleci (2), Jean-Patrick Chenu (3), \\ Jean-Rodolphe Vignes (2), Luc Bauchet (4), David Guiraud (1)
}

(1) INRIA, LIRMM, Montpellier, France; (2) Pellegrin Hospital, Bordeaux, France; (3) DETERCA, Bordeaux, France; (4) Gui de Chauliac Hospital, France

This article is distributed under the terms of the Creative Commons Attribution Noncommercial License (CC BY-NC 4.0) which permits any noncommercial use, distribution, and reproduction in any medium, provided the original author(s) and source are credited.

\begin{abstract}
Spinal cord injuries (SCI) result in the loss of movement and sensory feedback as well as organs dysfunctions. For example, nearly all SCI subjects loose their bladder control and are prone to kidney failure if they do not proceed to intermittent (self-) catheterization. Electrical stimulation of the sacral spinal roots with an implantable neuroprosthesis is a promising approach, with commercialized products, to restore continence and control micturition. However, many persons do not ask for this intervention since a surgical deafferentation is needed and the loss of sensory functions and reflexes become serious side effects of this procedure. Recent results renewed interest in spinal cord stimulation. Stimulation of existing pre-cabled neural networks involved in physiological processes regulation is suspected to enable synergic recruitment of spinal fibers. The development of direct spinal stimulation strategies aiming at bladder and bowel functions restoration would therefore appear as a credible alternative to existent solutions. However, a lack of suitable large animal model complicates these kinds of studies. In this article, we propose a new animal model of spinal stimulation -pig- and will briefly introduce results from one first acute experimental validation session.
\end{abstract}

Key Words: pig model, direct spinal electrical stimulation, bladder and bowel functions

Eur J Transl Myol 2016; 26 (2): 150-154

The National Spinal Cord Injury association estimates that 250,000 to 400,000 individuals living in the USA suffer from a spinal cord lesion. Extrapolating this statistic, there would be, at least 330,000 spinal cord injured (SCI) in the European Union and 1,300,000 worldwide. Lesions of the spinal cord results in sensorymotor deficits including intestinal and urinary tracts disorders. This is a major handicap for patient, who, despite of research efforts (stem cell, nerve growth factors, etc.), still cannot be cured. According to studies focused on SCI population quality of life, ${ }^{1}$ restoration of urinary and bowel functions remains of utmost importance, regularly quoted in patients top three main concerns.

Although already proposed as a solution to bladder hyperactivity, ${ }^{2}$ or for bladder emptying, existing functional electricalstimulation (FES) strategies remain insufficient. For hyperactivity, the Medtronic Interstim (Medtronic, USA) solution was implemented with implantation of epidural electrodes. Although source of symptoms reduction in case of an overactive bladder and minimally invasive, this device remain inappropriate in case of micturition problems. In the same way, the Finetech-Brindley stimulator (Finetech medical, UK) is indicated for complete spinal injured patient with incontinency problems. ${ }^{3}$ The necessary rhizotomies of the sensory dorsal sacral roots accompanying the implantation procedure, besides opening the reflex circuits at the spinal level (and thereby reduce spasticity of the urinary bladder) lead to a reduced lubrication in female patient or to a complete loss of reflex erection and ejaculation that, even if they are not very effective, are of great importance for male patients. ${ }^{4}$ Further side effects include lower limb contraction during the bladder emptying since the ventral sacral roots targeted by stimulation also include motor fibers of the lower leg muscles. Although effective, these clinical limitations tend to marginalize the use of this technology and procedure.

Despite these mixed results, the use of functional electrical stimulation strategies in visceral function rehabilitation should not be questioned but rather reoriented. Born in the beginning of the 1970s and originally associated with the treatment of chronic 


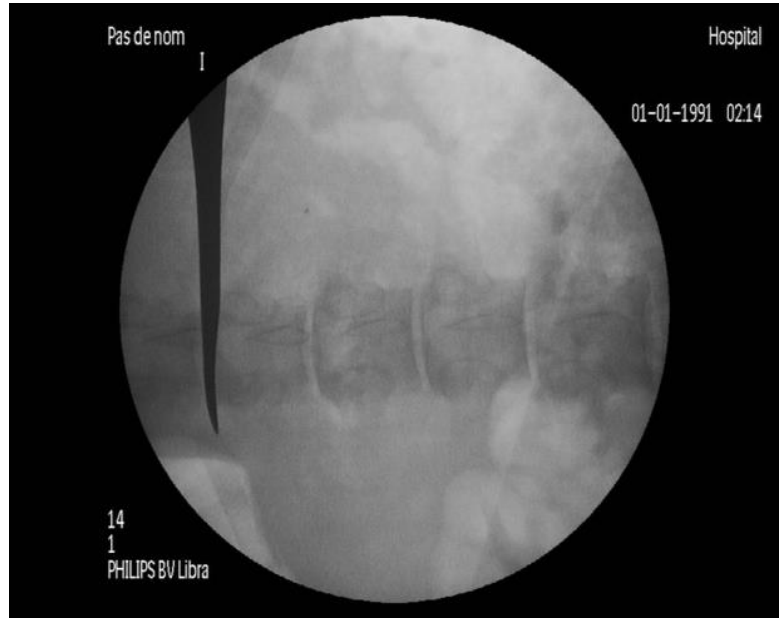

Fig 1: Identification of the thoraco-lumbar junction

neurological pain, ${ }^{5}$ epidural stimulation seems promising for new therapeutic strategies development. Primarily invested in the context of locomotion rehabilitation, epidural stimulation elicited both step-like EMG activity and locomotor synergies in patients lower limbs. ${ }^{6}$ These observations, further confirmed by numerous teams worldwide were subsequently enriched with new assumptions: postulates regularly affirming both training methods and afferent input involvement in functional rehabilitation. $^{7}$ The complexity of both lesional profiles, ethical issues and administrative procedures lead scientists to avoid human studies in benefits of small animal models (mice in most of cases) ${ }^{8}$ However, in spite of promising results, the transfer of knowledge from small animal model and the empirical aspect of the stimulation procedure have still to be overcome. Furthermore, the potential of this approach directly on urinary and bowel functions - in which potentiation by afferent output seems delicate - needs to be evaluated. Another important consideration is the necessary adaptation of technology to smaller animals.

We have worked on a large animal model - pig - to overcome size effects of rodent model and with the hope to facilitate the transfer of technological solutions and

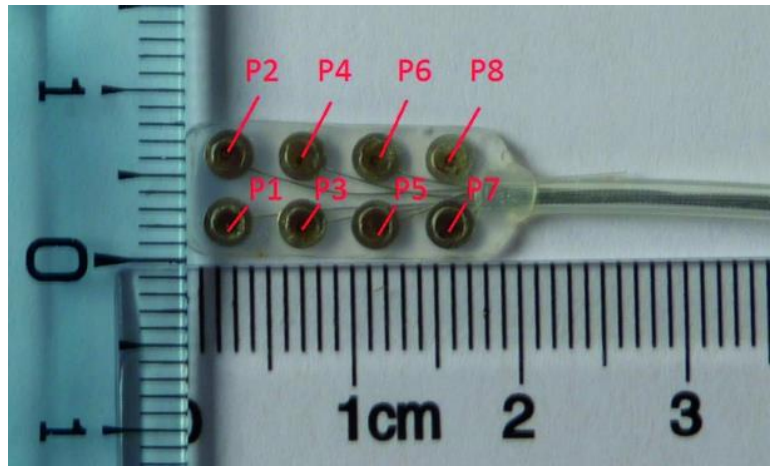

Fig 2: Matrix electrode dedicated to intradural stimulation, poles are labeled according to stimulation device connection

spatial selectivity results to human. We have targeted the pig spinal lower portion (L6-S4) and performed stimulation sessions, using a matrix electrode, in close contact with the spinal cord - intradural stimulation in order to facilitate functional mapping without current dissipation. The results mentioned in this paper are the foundation on which the future data processing algorithms will be built and which details will be presented during the conference.

\section{Materials and Methods}

Two objectives were pursued during this acute experiment - 1) validate a new animal model able to constitute a trade-off between small animal species and human for spinal cord stimulation purposes and 2) assess direct spinal stimulation impact on animal bladder and bowel functions - Accordingly, the implemented experimental protocol was preceded by a substantial literature study to identify a large animal model and define an anesthetic procedure preserving spinal cord excitability while minimizing animal suffering. Finally, experiment was facilitated by an innovative stimulation device developed within the team (CAMIN INRIA team, former DEMAR team, Montpellier, France.

Table 1: Stimulation session details

\begin{tabular}{cccccccc}
\hline Stimulation & Configurations & $\begin{array}{c}\text { Cathodic } \\
\text { pole }\end{array}$ & $\begin{array}{c}\text { Anodic } \\
\text { pole }\end{array}$ & $\begin{array}{c}\text { Frequency } \\
(\mathrm{Hz})\end{array}$ & $\begin{array}{c}\text { Pulse width } \\
(\mu \mathrm{s})\end{array}$ & $\begin{array}{c}\text { I initial } \\
(\mu \mathrm{A})\end{array}$ & $\begin{array}{c}\text { I final } \\
(\mu \mathrm{A})\end{array}$ \\
1 & 1 & 3 & 5 & 30 & 100 & 300 & 1600 \\
2 & 1 & 5 & 3 & 30 & 100 & 300 & 1600 \\
3 & 1 & 3 & 4 & 30 & 100 & 300 & 1600 \\
4 & 1 & 4 & 3 & 30 & 100 & 300 & 800 \\
5 & 1 & 4 & 6 & 30 & 100 & 300 & 800 \\
6 & 2 & 6 & 5 & 30 & 100 & 300 & 1500 \\
7 & 2 & 5 & 6 & 30 & 100 & 300 & 1500 \\
8 & 2 & 7 & 8 & 30 & 100 & 300 & 1000 \\
9 & 2 & 8 & 7 & 30 & 100 & 300 & 1200 \\
\hline
\end{tabular}




\section{An intermediate animal model of spinal cord stimulation}

Eur J Transl Myol 26 (2): 150-154

\section{A. Animal model identification: Urological,} neuroanatomical and anesthetic considerations

Beyond its wide availability, the porcine model especially for young individuals (3 to 4 months old) presents both urinary and spinal structures close to human ones. Presenting a bladder capacity of approximately $600 \mathrm{~mL}$ and an urethra of $8 \mathrm{~cm}$ long for $6 \mathrm{~mm}$ wide, $50 \mathrm{~kg}$ female individuals possess humanlike urinary characteristics. ${ }^{9}$ Unlike in males, where urethra presents many convolutions, females urinary tract is straight and joins the vaginal wall to half the distance of the cervix making transurethral catheterization possible. From a neuro-anatomical point of view, pig spinal characteristics have already been documented. Although stopping at S2 vertebra, pigs spinal cord possesses similarities with human structures (assumptions confirmed during surgery); similarities concerning both support and neural frameworks (vertebrae, meninges). ${ }^{10}$ Due to these peculiarities, studies dedicated to visceral centers identification within pig spinal grey matter have already been performed (mainly in minipigs). By highlighting cell bodies from (low) L6 to (up) S2, horseradish peroxidase injection indicated arrival of parasympathetic and somatic axons from bladder and external urethral sphincter (striated) to the lower portion of the spine. ${ }^{11}$ The combination of these points seem to justify the use of this animal species in studies dedicated to spinal cord stimulation, particularly regarding bladder and bowel functions restoration. While regularly use in experimental context, ${ }^{12}$ the elaboration of an anesthetic protocol compatible with both pig model and spinal stimulation was challenging. Given the identified constraints - 1) Adapted to porcine model, 2) long term efficiency (surgery + stimulation sessions), 3) Spinal excitability conservation, 4) urinary and bowel functions preservation. A combination of propofol and morphine (at relatively low doses) was retained to provide sufficient anesthesia

\section{B. Experimental procedure}

Acute experiment was performed on one Landras female pig (50 kg, 4 months old) according to European ethical rules concerning animal experiments (A2260 approval number). After premedication (intramuscular injection of Ketamine and Calmivet), the animal was placed under mechanical ventilation and an intravenous route was inserted at the ear vein for propofol and morphine delivery. To ensure animal welfare, both heart rate and temperature were monitored throughout the procedure.

After anesthesia induction, a transurethral catheter made of a silicon double-lumen bladder catheter was positioned and used to record lower urinary tract pressure (i.e. bladder response). Similarly, a rectal probe was placed to monitor changes in rectal pressure. Both pressures (bladder and rectal) were acquired using an acquisition system - developed within the team - triggered by stimulation. Prior neurosurgical exploration, radiographs were performed to identify the thoracolumbar junction (Fig. 1). Lumbar and sacral vertebrae were highlighted and an incision was performed in order to reveal L6 to S5 vertebral spinous process. A laminectomy was, then, performed and meninges were opened to expose the spinal cord.

\section{Experimental set-up}

For stimulation purposes, a matrix electrode (Axonic, France) was used to test different spatial configuration. Constituted by 8 contacts arranged in 4 rows of 2 poles, this array allowed test of several bipolar configurations (Fig. 2). Once the spinal cord exposed, the electrode was inserted between spinal tissue and S1 vertebral body - segment involved in urinary and

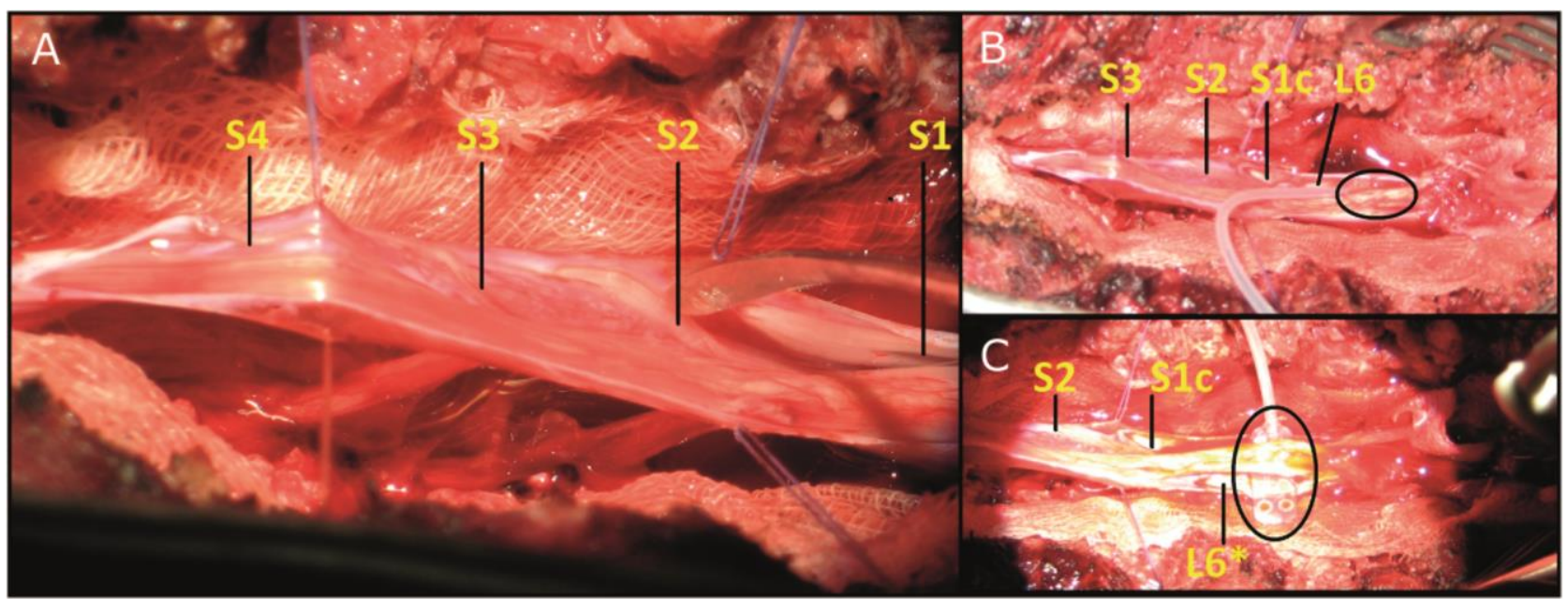

Fig 3. [A] Left: Exposure of the porcine spinal cord after meninges opening. [B] Top right: first electrode placement called configuration 1, [C] bottom right: second electrode placement named configuration 2. For both right pictures, electrode location is marked by an ellipse. * indicates a left spinal root. $c$ indicates the S1 right root cut for electrode placement. 


\section{An intermediate animal model of spinal cord stimulation}

Eur J Transl Myol 26 (2): 150-154

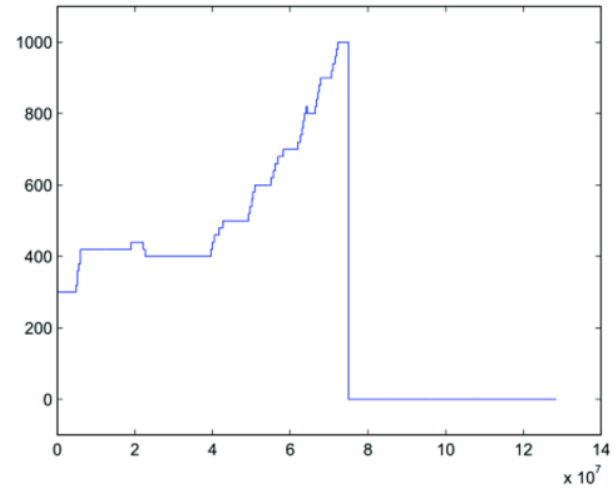

Fig 4. Stimulation intensity modulation based on the observed responses during the 8th stimulation (using a potentiometer). $x$-axis: time in $\mu$ s. $y$-axis: intensity in $\mu A$.

bowel function management according to literature - and bipolar stimulation were performed. Unfortunately, although intra-operative stimulation of the S1 right spinal root (S1) did not elicit any bladder or rectal responses, proximity between this root and spinal tissue induced mechanical constraints, thereby necessitating its section for electrode placement at the $\mathrm{S} 2$ ventral level. In addition to bladder and rectal pressures, a pair of needle electrode were inserted in the anal sphincter to monitor stimulation-induced EMG responses. These electrodes were connected to a differential EMG amplifier system (Biopac system, USA) and all signals (both pressure and EMG) were acquired using a data acquisition card (NI USB-6218, National Instrument, USA) connected to a personal computer equipped with Labview Signal Express software (National Instrument, USA) for data display and recording.

\section{Stimulation procedure}

To focus current on target tissue and avoid excessive dissipation an intradural approach was privileged. According to our knowledge, no pigs direct spinal stimulation was mentioned in literature, thus, particular attention was paid to bladder and bowel activation threshold determination.

The 12 current controlled channels stimulator - Stim'nD stimulation device developed within the team in cooperation with Axonic company (France) - used during the experiment enables modulation of the delivered current in real time (SENIS Manager software, CAMIN INRIA team, former DEMAR team, Montpellier, France). This allowed a graduate increase in stimulation intensity (20A minimum steps) and thus an identification of activation thresholds for all selected poles configurations (Fig. 4). Given the slow contraction dynamics of both bladder and rectum muscles, increases in intensity were performed in successive stimulation increments.

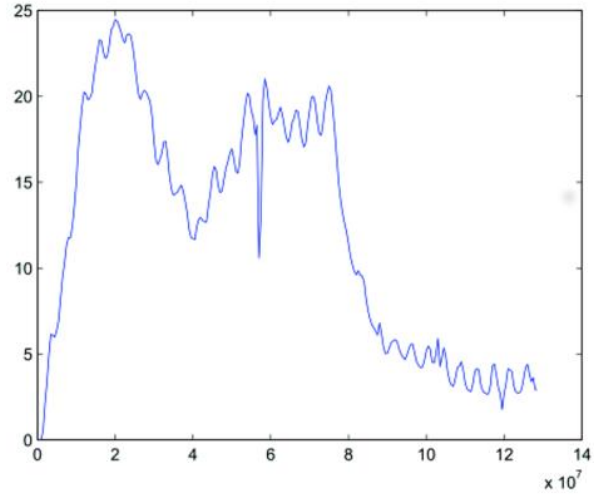

Fig 5. Bladder pressure recorded during the 8th stimulation. $x$-axis: time in $s$. y-axis: pressure in $\mathrm{cm} \mathrm{H} 2 \mathrm{O}$.

\section{Results}

A total of 9 stimulation sessions were performed for two different electrode positioning (see Fig. 3 and Table 1 for details). For each matrix placement, 4 monophasic stimulation sessions were devoted to study 2 distinct pairs of contacts, successively configured as anode/cathode and cathode/anode. These pairs of poles were chosen because of their close contact with the ventral spinal cord.

Although still in process, raw data show, as desired, the possible induction of bladder (see Fig. 5 for data corresponding to the 8th stimulation session) and rectal (Fig. 6, also recorded during the 8th stimulation) high responses - up to trigger stool evacuation (peroperating statement)- and suggest an interesting mapping implication of the cathode-anode distinction analysis. The observed spinal structures and pressure dynamics (a few tenth of $\mathrm{cmH} 2 \mathrm{O}$ ) seemed in agreement with those observed in human intraoperatively, thus positioning pig model as a perfect trade-off between rodent and human and confirming, at least partially, porcine model validity.

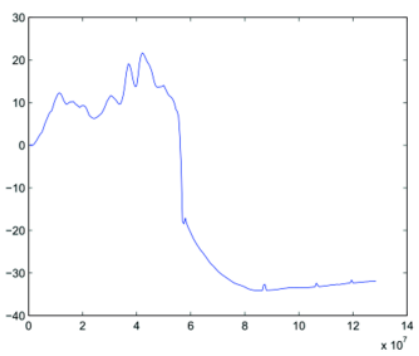

Fig 6. Rectal pressure recorded during the 8th stimulation. $x$-axis: time in $\mu$ s. $y$-axis: pressure in $\mathrm{cmH} 2 \mathrm{O}$. The final decrease is related to balloon ejection during bowel movements. 


\title{
An intermediate animal model of spinal cord stimulation
}

\author{
Eur J Transl Myol 26 (2): 150-154
}

\section{Discussion}

Even if data processing is, at present time, not finalized, the preliminary results confirmed, at least partially, the implemented methodology. Although performed on young - presenting doubtlessly a partially immature nervous system- and nonspinalized individuals (to avoid bias linked to this immaturity), the porcine model validity seems not have to be questioned. Similarly, the section of the S1 right spinal root can induce interrogation but its intraoperative monitoring seemed to exclude its involvement in lower visceral processes. From a technological point of view, matrix electrode design constitutes the limiting point for further selectivity studies. Nevertheless, the use of a powerful stimulation device enabling real time modulation of stimulation parameters allowed flexibility in threshold determination methodology. The observation of significant visceral responses tends to confirm the potential of spinal cord stimulation on urinary and intestinal functions. Other experiments were conducted recently to confirm these first results. Experiments whose data processing is expected for the next few weeks and results, we hope, ready for being presented and discussed during the IFESS conference.

\section{Contributions}

Thomas Guiho: Experiment preparation, data acquisition and processing, publication writing; Christine Azevedo Coste: Experiment preparation, Data acquisition, supervision of data processing and publication drafting, publication review; Claire Delleci: Urodynamic systems implementation and intraoperative monitoring, publication review; Jean-Patrick Chenu: Anesthetic procedure implementation and intraoperative animal welfare monitoring; Jean-Rodolphe Vignes: Experiment preparation, Neurosurgery, publication review; Luc Bauchet: Experiment preparation, Neurosurgery, publication review; David Guiraud: Experiment preparation, Data acquisition, supervision of data processing and publication drafting, publication review.

\section{Acknowledgement}

The authors wish to thanks all DETERCA staff. This work was realized in the context of the LabEx NUMEV program supported by the National Research Agency via the investment of the future program ANR-10-LABX20. Paper was prepared for the IFESS conference 2016.

\section{Conflict of Interest}

The author declare no conflict of interests.

\section{Corresponding Author}

Thomas Guiho, LIRMM - Université de Montpellier, Campus Saint Priest - Bat 5 - Bureau 03/234, 860 Rue de St Priest, 34095 Montpellier cedex 5 - France

E-mail: thomas.guiho@inria.fr

\section{E-mails of coAuthors}

Christine Azevedo Coste: azevedo@lirmm.fr

David Guiraud: david.guiraud@lirmm.fr

\section{References}

1. Anderson KD. Targeting recovery: priorities of the spinal cord-injured population. J Neurotrauma 2004;21:1371-83.

2. Amend B, Matzel KE, Abrams P, et al. How does neuromodulation work. Neurourol Urodyn 2011;30:762-5. doi: 10.1002/nau.21096. Epub 2011 Apr 1.

3. Brindley GS, Polkey CE, Rushton DN. Sacral anterior root stimulators for bladder control in paraplegia. Spinal Cord 1982;20:365-16.

4. Vignes JR, De Seze M, Sesay M, et al. Neurostimulation des racines sacres antrieures avec rhizotomies postrieures (technique de brindley). Neuro-chirurgie 2003;49:383-11.

5. Illis LS, Sedgwick EM, Oygar AE, Awadalla MS. Dorsal-column stimulation in the rehabilitation of patients with multiple sclerosis. The Lancet 1976; 307:1383-3.

6. Dimitrijevic MR, Gerasimenko Y, Pinter MM. Evidence for a spinal central pattern generator in humans. Annals of the New York Academy of Sciences 1998; 860:360-16.

7. Harkema S, Gerasimenko Y, Hodes J, et al. Effect of epidural stimulation of the lumbosacral spinal cord on voluntary movement, standing, and assisted stepping after motor complete paraplegia: a case study. The Lancet 2011;377:1938-9.

8. Van den brand R, Heutschi J, Barraud Q, et al. Restoring voluntary control of locomotion after paralyzing spinal cord injury. Science 2012;336:1182-3.

9. Dass N, McMurray G, Greenland JE, A. Brading F. Morphological aspects of the female pig bladder neck and urethra: quantitative analysis using computer assisted 3-dimensional reconstructions. J Urol 2001; 165: 1294-5.

10. Pleticha J, Maus TP, Jeng-Singh C, et al. Pig lumbar spine anatomy and imaging-guided lateral lumbar puncture: A new large animal model for intrathecal drug delivery. J Neurosci Methods 2013;216:10-5. doi: 10.1016/j.jneumeth.2013.03 .006. Epub 2013 Mar 19

11. Kuipers R, Izhar Z, Gerrits PO, et al. Location of bladder and urethral sphincter motoneurons in the male guinea pig (cavia porcellus). Neurosci Lett 2004;362:57-4.

12. Kaiser GM, Heuer MM, N. R. Frühauf, et al. General handling and anesthesia for experimental surgery in pigs. J Surg Res. 2006;130:73-9. Epub 2005 Nov 14. 